\title{
A CASE OF A YOUNG WOMAN WITH CERVICO-MEDIASTINAL MALIGNANT GOITER
}

\author{
G. Yankov', P. Gecov², M. Kovacheva³ , E. Mekov', R. Petkov', B. Vladimirov³ \\ ${ }^{1}$ Department of Respiratory Diseases, Medical Faculty, Medical University - Sofia, Bulgaria \\ ${ }^{2}$ Department of Radiology, Medical Faculty, Medical University - Sofia, Bulgaria \\ ${ }^{3}$ Department of Gastroenterology, Medical Faculty, Medical University - Sofia, Bulgaria
}

\begin{abstract}
The main treatment in nearly every case of thyroid cancer is surgery. The exception often refers to anaplastic carcinoma because this cancer is already widespread at the time of diagnosis. Most of the cases are treated with thyroidectomy, but small tumors that spread inside the thyroid gland might be treated by lobectomy. Intrathoracic goiter accounts for $5.8 \%$ of all mediastinal masses. On the other hand, the incidence of thyroid malignancy in cervico-mediastinal thyroid masses is $7,7 \%$. In such cases, total thyroidectomy with en block removal of the mediastinal portion of the gland is the treatment of choice. We present a case of a 34-year-old woman with cervico-mediastinal malignant goiter.
\end{abstract}

Key words: goiter, cervico-mediastinal lesion, total thyroidectomy

Corresponding author: Evgeni Mekov, MHATPD "Sveta Sofia", 19, "Acad. Ivan Geshov" blvd, 1431 Sofia, Bulgaria, tel. +359 888320 476, e-mail: evgeni.mekov@gmail.com

\section{INTRODUCTION}

$\mathrm{T}$ he main treatment in nearly every case of thyroid cancer is surgery. The exception often refers to anaplastic carcinoma, because this cancer is already widespread at the time of diagnosis. Most of the cases are treated with thyroidectomy, but small tumors that spread inside the thyroid gland might be treated by lobectomy. Intrathoracic goiter accounts for $5.8 \%$ of all mediastinal masses. On the other hand, the incidence of thyroid malignancy in cervico-mediastinal thyroid masses is $7,7 \%$. In such cases, total thyroidectomy with en block removal of the mediastinal portion of the gland is the treatment of choice. We present a case of a 34-year-old woman with cervico-mediastinal malignant goiter.

\section{CASE REPORT}

A 34-year-old woman was admitted to the Thoracic Surgery Department with complaints of pain and tight- ness in the neck and behind the sternum, which have gradually been worsening for 2 years, and hoarseness for 6 months. She was diagnosed with thyroid papillary carcinoma from fine-needle biopsy, but for about 2 years the patient was monitored and referred to several surgical institutions without performing surgical intervention because she was considered a high-risk patient. The thyroid gland was enlarged with left lobe formation with a lobulated surface, denseelastic consistency, poor mobility, and was painless on palpation (Fig. 1). The rest of the physical exam was unremarkable. She denied any concomitant diseases. FT3, FT4, and TSH levels were normal, as were other blood and biochemical parameters.

Thoracic ultrasound confirmed a large left-sided tumor formation in the upper and anterior mediastinum, originating from the left lobe of the thyroid gland. The formation was with dimensions $55 / 59 \mathrm{~mm}$, cystically altered. Neovascularization was also found. A sec- 
ond formation in the anterior mediastinum in front of the aortic arch, next to the right pulmonary artery measuring 58/46 $\mathrm{mm}$ was also found. The left brachiocephalic vein was fibrotic, reduced in size, and compressed. The superior vena cava demonstrated preserved blood flow. The second formation in the upper and anterior mediastinum had preserved pulsating mobility relative to the aortic arch and was displacing the left subclavian artery laterally, while the left common carotid artery was passing through the formation (Fig. 2). Fine-needle aspiration biopsy of the left thyroid lobe confirmed papillary carcinoma.

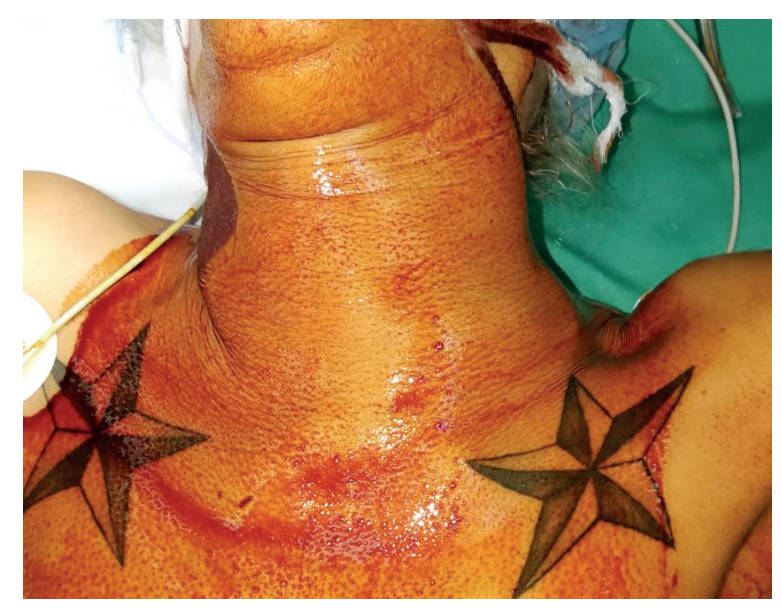

Fig. 1. The patient before the intervention

Fibrobronchoscopy showed left vocal cord paresis. The trachea was slightly dislocated to the right but showed no significant infiltration. The carina and the two main bronchi were intact. Fibroesophagoscopy confirmed a normal esophageal mucosa with leftsided external compression.

The CT scan (Fig. 3) revealed an enlarged thyroid gland mainly at the expense of its left lobe. Hy- podense nodules were found in its structure. At the level of the upper thoracic aperture and the upper anterior mediastinum, numerous lesions of various calibers with a heterogeneous structure, with the presence of solid and cystic components and calcifications were found. After i.v. contrast application the nodules increased their density inhomogeneously. The described formations were located around the vessels and trachea, pushing the supra-aortic vascular structures and the trachea to the right without engaging them. The image revealed the presence of right innominate vein stenosis.

Kocher cervicotomy was performed. A nodally altered left lobe of the thyroid gland with dimensions $30 / 30 \mathrm{~mm}$ was reached. Laterally, another denseelastic tumor formation about $90 / 40 \mathrm{~mm}$ in size was detected towards the left supraclavicular region and descending to the upper mediastinum. The incision had been expanded to T-shaped and a midline sternotomy was performed. A third formation was loosely connected to the second with a size of $80 / 40 \mathrm{~mm}$ located caudally from the left lobe of the thyroid gland which was descending retrosternal, reaching the aortic arch between the brachiocephalic trunk and carotid artery and pushing the trachea and esophagus to the right. The left common carotid artery, including its bifurcation, was covered as a cuff by the described cervicomediastinal formation, without infiltration. The involuted thymus gland was mobilized with the surrounding adipose tissue and separated from the pericardium. The brachiocephalic trunk, the left carotid artery, the left jugular vein, and the left vagus nerve were taken on vascular tourniquets. An isthmotomy was performed, the left thyroid gland was mobilized and resected. An unaltered pretracheal lymph node was removed. The left brachiocephalic vein was dissected along its entire length. With acute dissection,
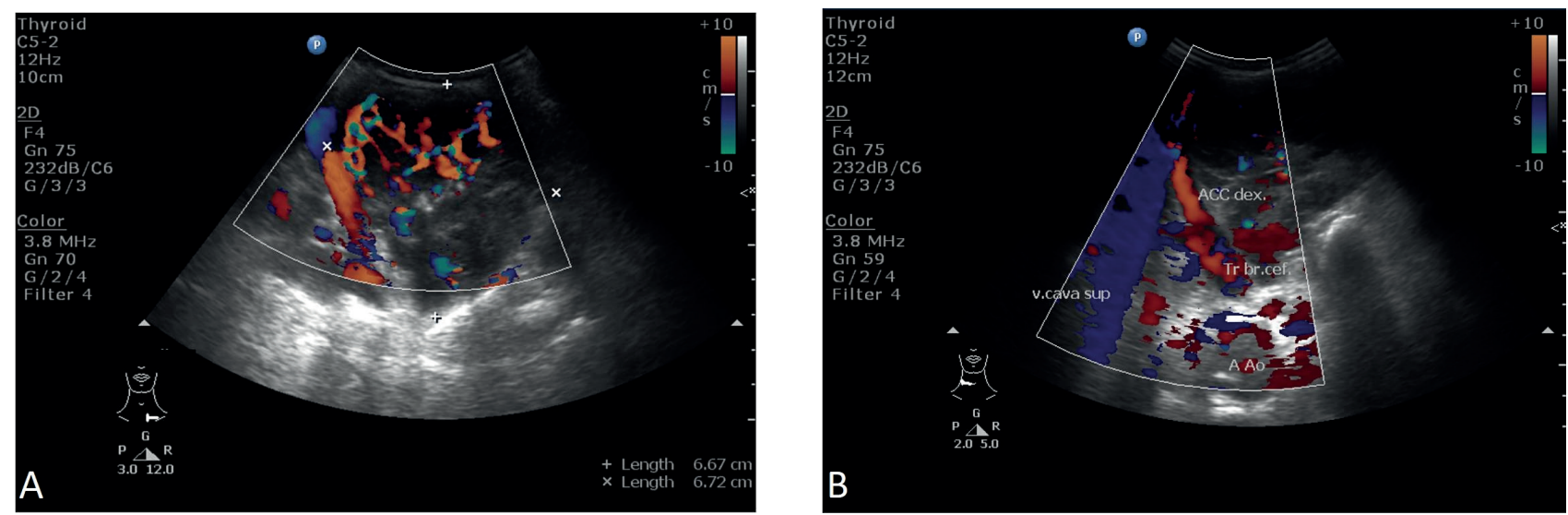

Abbr.: V. cava sup. - Superior vena cava; ACC dex. - right common carotid artery; Tr. br. cef. - truncus brachiocephalicus; A. Ao. - ascending aorta.

Fig. 2. Ultrasound view of the thyroid gland. A - left lobe; B - right lobe 
the second formation, descending caudally from the left lobe and reaching the angle between the left common carotid artery and the brachiocephalic trunk and dorsally from the left brachiocephalic vein was mobilized. The terminal part of the ductus thoracicus in the area of its infusion into the left venous angle was visualized. Several macroscopically unaltered lymph nodes located behind the left carotid artery and the left jugular vein, around the left phrenic nerve, as well as lymph nodes behind and around the superior vena cava, were removed. The third formation was sharply separated from the aortic arch, the left pulmonary artery, and in the proximal direction the left innominate vein was brought behind the left vein to the neck, and the described cervicomediastinal formation was extirpated. A total thyroidectomy was performed with the removal of the right lobe of the thyroid gland (Fig.4).

Macroscopically the first circular encapsulated formation was 91/67/30 $\mathrm{mm}$ in size. Two larger and several smaller cysts filled with necrotic contents and hemorrhages were found on a dissection. A second encapsulated formation was irregularly shaped and $88 / 39 / 21 \mathrm{~mm}$ in size (Fig. 5). The dissection showed a nodular appearance and many necrotic areas, blood, and calcifications.

Histological examination of the first circular encapsulated tumor formation was positive for papillary thyroid carcinoma with a maximum size of $3 \mathrm{~cm}$, reaching close to the capsule without engaging it, without residual gland parenchyma or lymphoid tissue, severe secondary degenerative changes - cystic transformation, fibrosis, fresh and old hemorrhages with an abundance of hemosiderophages and numerous psammoma bodies; five lymph nodes with reactive changes, without metastases. In the second encapsulated material, tumor parenchyma of papillary thyroid carcinoma was found, encapsulated with a dense fibrous capsule, without residual thyroid parenchyma or lymphoid tissue; four lymph nodes without metastases.
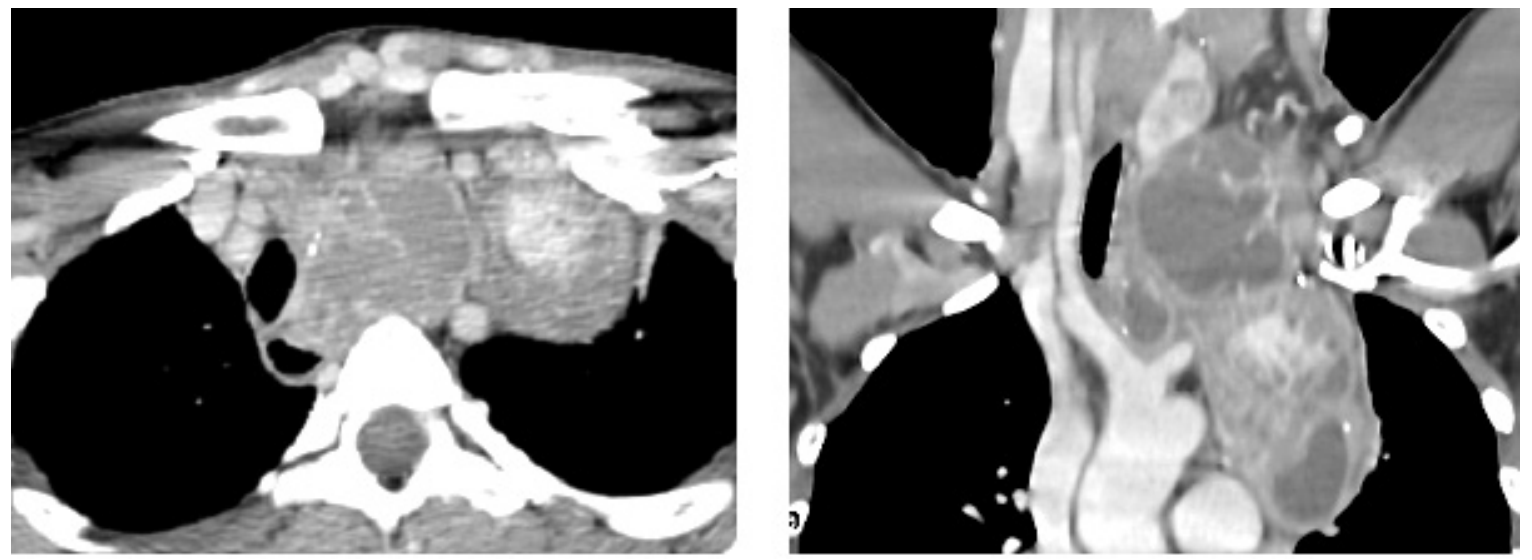

Fig. 3. Computed tomography of the thyroid gland. A - axial image; $\mathbf{B}$ - coronal reconstruction
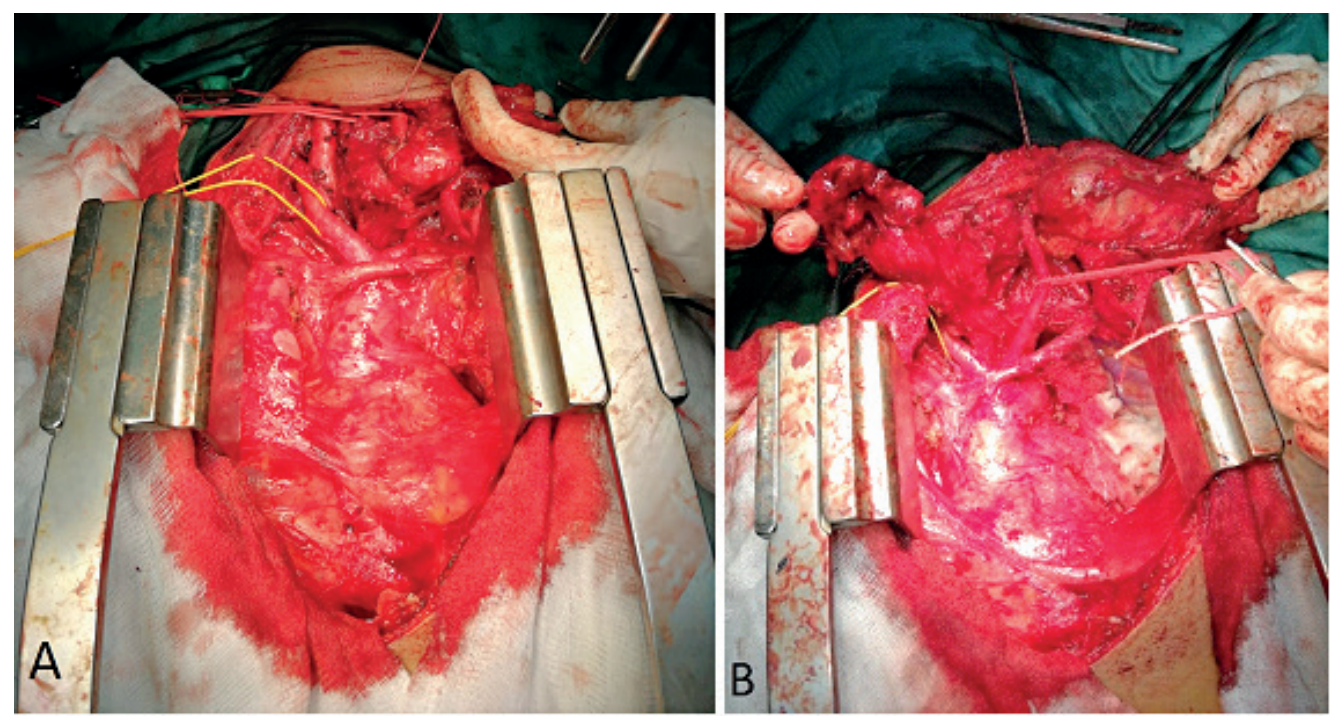

Fig. 4. A - cervicotomy continued to sternotomy; B - a view after mobilization and extirpation of the formations 


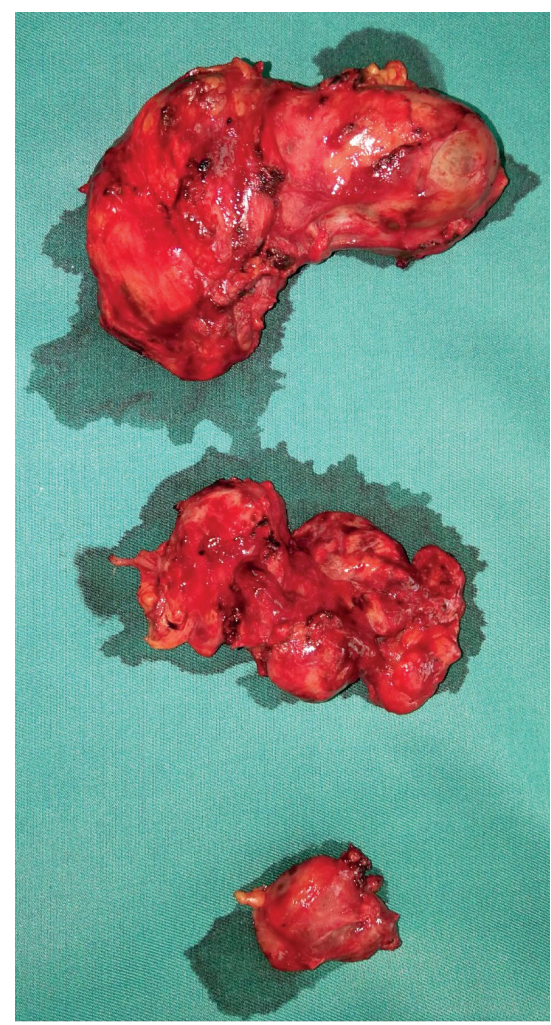

Fig. 5. Macroscopic specimens

Histological examination of the right lobe of the thyroid gland revealed colloidal nodular goiter. The lymph nodes from the isthmus of the thyroid gland, left vascular bundle of the neck, retro- and paracaval, and around the left phrenic nerve were without metastases.

The conclusion from the pathohistological examination was: papillary carcinoma of the thyroid gland, involving the left lobe of the gland $(30 \mathrm{~mm}$ in diameter) and two separate mediastinal formations with a diameter of $90 \mathrm{~mm}$ (in mediastinal micro- and macrofollicular colloid goiter); no metastases were found in 14 lymph nodes.

The postoperative period was uneventful and the patient was discharged. There was no relapse after a two-year follow-up period.

\section{DISCUSSION}

Nearly $85-95 \%$ of mediastinal thyroid formations are benign and occur as a result of iodine deficiency [1]. The majority of mediastinal goiters are diagnosed in the sixth decade of life, more often in women than in men (3:1) $[2,3]$. Thirty-eight percent $(38 \%)$ of goiters are located in the prevascular region, $33 \%$ - between the vessels and the trachea, and $27 \%$ - behind the trachea [4]. Papillary thyroid cancer generally has a good prognosis, with a slow progression and relatively late metastases.

The diagnostic work-up includes clinical and imaging studies and is completed by fine-needle aspiration biopsy [5]. The presence of lymph node metastases has little effect on the overall survival, although it does affect relapses [6]. Early diagnosis and surgical treatment reduce metastases and local recurrences [7].

Malignant degeneration of cervical mediastinal goiters occurs in 7.7\% [8]. Moreover, extended surgical access as in the presented case is required in only $2-3 \%$ of patients in cases with intrathoracic vessel involvement, displacement, or compression of the aortic arch, vena cava superior syndrome, or malignant transformation [9]. In the presented case we observed malignancy of cervicomediastinal goiter. She was successfully operated, despite the intraoperative difficulties in isolating the thyroid mass. Thyroidectomy was performed by combined cervicosternotomy, without which it would not be possible to control critical main mediastinal and cervical blood vessels.

\section{CONCLUSION}

Early surgical treatment of malignant cervical mediastinal goiter is extremely important. It contributes to favorable long-term results.

Disclosure summary: The authors have nothing to disclose.

\section{REFERENCES}

1. Aghajanzadeh M, Asgary MR, Mohammadi F, et al. An investigation into symptoms, diagnosis, treatment, and treatment complications in patients with retrosternal goiter. J Family Med Prim Care 2018;7(1):224-9.

2. Huang WC, Huang $\mathrm{CH}$, Hsu $\mathrm{HC}$, et al. Intrathoracic Goiter in Elderly Patients. International Journal of Gerontology. 2013;7(1):8-12.

3. Tsilimigras DI, Patrini D, Antonopoulou A, et al. Retrosternal goitre: the role of the thoracic surgeon. J Thorac Dis 2017;9(3):860-3.

4. de Perrot M, Fadel E, Mercier O, et al. Surgical management of mediastinal goiters: when is a sternotomy required? ThoracCardiovasc Surg 2007;55(1):39-43.

5. Lee EW, Chen C, Sauk S, Ragavendra N. How diagnostic is ultrasound-guided neck mass biopsy (fine-needle capillary sampling biopsy technique)? evaluation of 132 nonthyroid neck mass biopsies with pathologic analysis over 7 years at a single institution. Journal of Ultrasound in Medicine 2009;28(12):1679-84.

6. Tanaka K, Sonoo H, Hirono M, et al. Retrospective analysis of predictive factors for recurrence after curatively resected papillary thyroid carcinoma. Surgery Today 2005;35(9):714-9.

7. Spartalis ED, Karatzas T, Charalampoudis P, Vergadis C, Dimitroulis D. Neglected papillary thyroid carcinoma seven years after initial diagnosis. Case reports in oncological medicine 2013:148973.

8. Di Crescenzo V, Vitale M, Valvano L, et al. Surgical management of cervico-mediastinal goiters: our experience and review of the literature. International Journal of Surgery 2016;28(Suppl 1):S47-S53.

9. Zhao H, Ren D, Liu Y, et al. Complete transthoracic resection of giant posterior mediastinal goiter: case report and review of surgical strategies. Onco Targets Ther 2016;9:2415-9.

Received: 08 September 2020, Accepted: 08 October 2020 